\title{
ON THE SUMMABILITY OF A CERTAIN CLASS OF SERIES OF JACOBI POLYNOMIALS*
}

\author{
BY A. P. COWGILL
}

1. Introduction. The result obtained in this paper is as follows:

The series

$$
\sum_{n=1}^{\infty} n^{i} \frac{(p+1)(p+3) \cdots(p+2 n-1)}{2^{n} n !} X_{n}^{(p-1) / 2}(x),
$$

where $X_{n}{ }^{(p-1) / 2}(x)$ (hereafter indicated simply by $X_{n}$ ) is a symmetric Jacobi polynomial, $\uparrow p>-1$, and $i$ a positive integer, is summable $(C, k), k>i-1 / 2$, for the range $-1<x<1$.

The proof is limited to symmetric Jacobi polynomials because of the necessity of having the recursion formula $\ddagger$ of first degree in $n$. Unless explicitly stated otherwise, $x$ is confined to the range $-1<x<1$, and $p>-1$, throughout this paper.

In the proof the sum of the $n$ first terms of the given series is transformed, following the method employed by Brenke for Hölder summability of certain series of Legendre polynomials, $\S$ by the recursion formula for Jacobi polynomials into a new sum of $n$ terms, plus four additional terms. Then convergence factors for summability $(C, i-1)$ are applied, followed by those of summability $(C, j), j>1 / 2$, necessary to evaluate the additional

* Presented to the Society, November 30,1934 . This paper is a portion of a thesis presented to the Faculty of the Graduate College, The University of Nebraska.

$\dagger$ This is denoted by $F(-n, n+p,(p+1) / 2,(1-x) / 2)$ in the notation of Darboux, Mémoire sur l'approximation des fonctions de très grands nombres, Journal de Mathématiques, (3), vol, 4 (1878), pp. 5-60, 377-416; p. 22. It is $G_{n}(p,(p+1) / 2,(1-x) / 2)$ in the notation of $\mathrm{R}$. Courant and D. Hilbert, Methoden der Mathematischen Physik, vol. 1, p. 74. It is $X_{n}{ }^{((p-1) / 2,(p-1) / 2)}(x)$ in the notation of G. Pblya and G. Szegö, Aufgaben und Lehrsätze aus der Analysis, vol. 2, pp. 93-94, where the orthogonality property is expressed by means of the equality $\int_{-1}^{1}(1-x)^{(p-1) / 2}(1+x)^{(p-1) / 2} X_{n}((p-1) / 2,(p-1) / 2) X_{m}((p-1) / 2,(p-1) / 2) d x$ $=0(m \neq n ; m, n=0,1, \cdots)$.

$\ddagger$ Darboux, loc. cit., p. 378.

$\S \mathrm{W}$. C. Brenke, On the summability and generalized sum of a series of Legendre polynomials, this Bulletin, vol. 39 (1933), pp. 821-824. 
terms involving $n$, making the equivalent of summability $(C, k), k>i-1 / 2$. The first application of summability causes the highest ordered part of the sum of the two additional terms involving $n$ to take the form of a series, which is equal to the Cauchy product of two series, one of which is summable to a finite value and the other to the value zero.

Certain well known theorems of summability are used.*

THEOREM 1. If two series are respectively summable $(C, \alpha)$ and $(C, \beta)$, their Cauchy product is summable $(C, \alpha+\beta+1)$ to the product of the sums of the series.

Theorem 2. If $\sum_{n=0}^{\infty} u_{n}$ is summable $(C, \delta)$ to the value $s$, this implies that $\lim _{x \rightarrow 1-0} \sum_{n=0}^{\infty} u_{n} x^{n}=s$.

Theorem 3. A series that is bounded $(C, \alpha), \alpha \geqq-1$, is summable $(C, \delta>\alpha)$ with the sum $s$, if $\lim _{x \rightarrow 1-0} \sum_{n=0}^{\infty} u_{n} x^{n}=s$.

Theorem 4. The summability $(C, \delta)$ of $\sum u_{n}$ implies $u_{n}=o\left(n^{\delta}\right)$ and $s_{n}=o\left(n^{\delta}\right)$.

THEOREM 5. The existence of $\lim _{n \rightarrow \infty} s_{n}^{(\delta+\gamma)}=s$, where $s_{n}^{(\delta+\gamma)}$ denotes the Cesàro nth partial mean of order $\delta+\gamma$, implies that of the double mean $S_{n}{ }^{(\delta, \gamma)}$ of orders $\delta$ and $\gamma$ :

$$
\lim _{n \rightarrow \infty} S_{n}^{(\delta, \gamma)}=\lim _{n \rightarrow \infty} S_{n}^{(\gamma, \delta)}=\lim _{n \rightarrow \infty} s_{n}^{(\delta+\gamma)},
$$

and vice versa, provided $\delta, \gamma, \delta+\gamma>-1$.

2. The Polynomial $X_{n}$. The recursion formula is $\dagger$

$$
x X_{n}=\frac{n+p}{2 n+p} X_{n+1}+\frac{n}{2 n+p} X_{n-1} .
$$

The generating function, $\ddagger$ when

$$
\begin{aligned}
a_{n} & =\frac{(p+1)(p+3) \cdots(p+2 n-1)}{2^{n} n !}=\frac{\Gamma((p+1) / 2+n)}{\Gamma((p+1) / 2) \Gamma(n+1)} \\
& =O\left(n^{(p-1) / 2}\right),
\end{aligned}
$$

* E. Kogbetliantz, Mémorial des Sciences Mathématiques, vol. 51, pp. 19$20,29,37,30-31$, and 23 , respectively.

$\dagger$ Darboux, loc. cit., p. 378.

$\ddagger$ Darboux, loc. cit., p. 23. 


$$
\begin{array}{ll}
\frac{\left(\left(1-x^{2}\right) / 4\right)^{(1-p) / 2}\left[2 t x-2+2\left(1-2 t x+t^{2}\right)^{1 / 2}\right]^{(p-1) / 2}}{(2 t)^{p-1}\left(1-2 t x+t^{2}\right)^{1 / 2}} & \\
=\sum_{r=0}^{\infty} a_{r} X_{r} t^{r}, & (0<t<1) .
\end{array}
$$

I find by the method used for Legendre polynomials by Byerly* that $X_{r}$ in the following formula

$$
\sum_{r=0}^{\infty} \frac{\Gamma(r+p)}{\Gamma(p) \Gamma(r+1)} X_{r} t^{r}=\frac{1}{\left(1-2 t x+t^{2}\right)^{p / 2}}, \quad(0<t<1),
$$

satisfies the Jacobi recursion formula (1). We have also

$$
\begin{aligned}
X_{n} & =O\left(n^{-p / 2}\right), \quad(-1<x<1) . \dagger \\
X_{n}(1) & =1 . \ddagger
\end{aligned}
$$

3. Transformation of the Series. Multiplying (1) by $c_{n}$ and summing from 1 to $n$, we get

$$
\begin{aligned}
x \sum_{r=1}^{n} c_{r} X_{r}= & \sum_{r=1}^{n} \frac{r+p}{2 r+p} c_{r} X_{r+1}+\sum_{r=1}^{n} \frac{r}{2 r+p} c_{r} X_{r-1} \\
= & \sum_{r=1}^{n}\left[\frac{r-1+p}{2 r-2+p} c_{r-1}+\frac{r+1}{2 r+2+p} c_{r+1}\right] X_{r} \\
& -c_{0} X_{1}+\frac{n+p}{2 n+p} c_{n} X_{n+1}+\frac{1}{2+p} c_{1} X_{0} \\
& -\frac{n+1}{2 n+2+p} c_{n+1} X_{n} .
\end{aligned}
$$

If $c_{n}=n^{i} a_{n}$, the coefficient of $X_{r}$ in (4) takes the form

$$
U_{r} \equiv\left[r^{i}+b_{1}(p) r^{i-2}+b_{2}(p) r^{i-3}+\cdots\right] a_{r},
$$

where the coefficient of $a_{r}$ is a polynomial in descending powers of $r$. The last term in the square brackets will be of order $O\left(n^{\delta}\right)$,

* W. E. Byerly, Fourier Series, 1893, p. 151. See also N. Nielsen, Théorie des Fonctions Métasphériques, 1911.

$\dagger$ Darboux, loc. cit., p. 378.

$\ddagger$ This is shown by using equation (1), Darboux, loc. cit., p. 377, and making the transformation $x=(1-\xi) / 2, x=0$ corresponding to $\xi=1$. 
$\delta \leqq-1$, which, when multiplied by $a_{r} X_{r}$ and summed, will give a convergent series, for

$$
a_{r} X_{r} O\left(n^{\delta}\right)=O\left(n^{(p-1) / 2-p / 2+\delta}\right)=O\left(n^{\delta^{\prime}}\right),
$$

where $\delta^{\prime}=\delta-1 / 2<-1$.

The terms in (4) free from $n$ are

$$
R_{0} \equiv-c_{0} X_{1}+\frac{1}{2+p} c_{1} X_{0} \text {. }
$$

These carry over without change in the process of summation.

The remainder terms in (4) will be

$$
R_{n} \equiv \frac{n+p}{2 n+p} c_{n} X_{n+1}-\frac{n+1}{2 n+2+p} c_{n+1} X_{n} .
$$

The relation (4) can now be written in the form

$$
x \sum_{r=1}^{n} c_{r} X_{r}=R_{0}+\sum_{r=1}^{n} U_{r} X_{r}+R_{n}, \quad\left(c_{r}=r^{i} a_{r}\right) .
$$

4. Application of Summation $(C, k)$ to (6). Apply Cesàro summation of order $k$ to both sides of (6). Representing by $S_{n, i}^{(k)}$ the $k$ th Cesàro mean of $S_{n, i}=\sum_{r=0}^{n} r^{i} a_{r} X_{r}$, we find after transposing the sum $S_{n, i}^{(k)}$ from the right to the left side of equation (6),

$$
\begin{aligned}
& (x-1) S_{n, i}^{(k)}=R_{0}+b_{1}(p) S_{n, i-2}^{(k)}+\cdots+b_{i-1}(p) S_{n, 0}^{(k)} \\
& \quad+\left[S_{n}^{(k)} \text { of a convergent series }\right]+(C, k) \text { of } R_{n} .
\end{aligned}
$$

The order of the terms of $R_{n}$ is $O\left(n^{i-1 / 2}\right)$, so, by Theorem 4 , Cesàro summability of order $<i-1 / 2$ could not be expected.

Then, applying Cesàro summation of order $k$, so chosen that $(C, k) R_{n} \rightarrow 0$ as $n \rightarrow \infty$, and writing $\lim _{n \rightarrow \infty} S_{n, k}^{(k)}=S_{\infty, h}^{(k)}$, we have $S_{\infty, i}^{(k)}$ expressed in terms of $R_{0}, S_{\infty, i-2}^{(k)}, S_{\infty, i-3}^{(k)}, \cdots, S_{\infty, 0}^{(k)}$ and a convergent series. Values of $S_{\infty, i}^{(k)}$ must be calculated successively; we begin with $S_{\infty, 0}^{(k)}$, which can be obtained from the generating function (2), and take successive integral values of $i$, beginning with $i=1$. As stated in the introduction, two applications of Cesàro summation, which are equivalent to summation $(C, k)$, are then used to make $(C, k) R_{n} \rightarrow 0$ as $n \rightarrow \infty$. 
5. Summation $(C, i-1)$ Applied to $R_{n}$. The convergence factors for summability $(C, k)$ have the form

$$
\frac{\Gamma(k+n-r+1)}{n^{k} \Gamma(n-r+1)},
$$

where $n$ represents the total number of terms in the sum under consideration and $r$ the rank of the particular term to which the convergence factor is applied. One groups the remainder $R_{n}$ with the $n$th term of the sum in the right-hand member of (6) so that the $n$th Cesàro convergence factor will be applied to $R_{n}$ as well as to $U_{n} X_{n}$. The remainder $R_{n}$ becomes, after application of summation $(C, i-1)$,

$$
\begin{aligned}
& R_{n}^{(i-1)}=\Gamma(i)\left[\frac{(n+p) n}{2 n+p} X_{n+1}\right. \\
& \left.-\frac{(n+1)^{i}}{(2 n+2+p)} \frac{(p+2 n+1)}{2 \cdot n^{i-1}} X_{n}\right] a_{n} \text {, } \\
& \text { (8) } R_{n}^{(i-1)}=\frac{1}{2} a_{n} \Gamma(i)\left[1+O\left(\frac{1}{n}\right)\right](n+p)\left(X_{n+1}-X_{n}\right) \text {. }
\end{aligned}
$$

To evaluate this expression as $n \rightarrow \infty$, the Christoffel-Darboux identity is used.

6. Use of the Christoffel-Darboux Identity. This identity, when modified to conform to the notation of this paper, ${ }^{*}$ becomes

$$
\begin{aligned}
& \frac{\Gamma(n+p)}{\Gamma(p) \Gamma(n+1)}(n+p) \frac{X_{n+1}-X_{n}}{x-1} \\
& \quad=\sum_{r=0}^{n} \frac{\Gamma(r+p)}{\Gamma(p) \Gamma(r+1)}(2 r+p) X_{r} .
\end{aligned}
$$

Differentiate both sides of (3), multiply throughout by $2 t$, and add to (3); we get

$$
\sum_{r=0}^{\infty} \frac{\Gamma(r+p)}{\Gamma(p) \Gamma(r+1)}(2 r+p) X_{r} t^{r}=\frac{p\left(1-t^{2}\right)}{\left(1-2 t x+t^{2}\right)^{p / 2+1}},
$$

* Darboux, loc. cit.; one substitutes (44), p. 46, in (14), p. 381. After changing $x$ and $z$ into $(1-x) / 2$ and $(1-z) / 2$, respectively, let $\alpha=p$ and $\gamma=(p+1) / 2$. Then let $z=1$, so that $Z_{n}=Z_{n+1}=1$, and simplify. 
which one can write in the form

$$
\begin{aligned}
& \sum_{r=0}^{\infty} \frac{\Gamma(r+p)}{\Gamma(r+1)}(2 r+p) X_{r} t^{r} \\
& \quad=\frac{\Gamma(p+1)}{\left(1-2 t x+t^{2}\right)^{p / 2}} \frac{1-t^{2}}{1-2 t x+t^{2}},
\end{aligned}
$$

which incidentally checks with well known relations for Tchebychef (trigonometric) polynomials, where $p=0$, and Legendre polynomials, where $p=1$.

From an article by Fejér,* we have

$$
\frac{1-t^{2}}{1-2 t x+t^{2}}=2\left(\frac{1}{2}+\sum_{r=1}^{\infty} t^{r} \cos r v\right), \quad(x=\cos v),
$$

which is the generating function of the trigonometric polynomials. Chapman proved $\dagger$ that $\left(1 / 2+\sum_{r=1}^{\infty} \cos r v\right)$ is summable $(C, k)$, $k>0$, to the value zero.

To obtain the order of summability of

$$
\sum_{r=0}^{\infty} \frac{\Gamma(r+p)}{\Gamma(p) \Gamma(r+1)} X_{r}
$$

(which is formula (3) for $t=1$ ), we may use the method of proof given by Chapman $\ddagger$ for Legendre polynomials, based on obtaining an asymptotic expression for $S_{n}^{(k)}$ for the above series by the method of Darboux. Let $x=\cos \theta$. Since

$$
\frac{1}{\left(1-2 z \cos \theta+z^{2}\right)^{p / 2}}=\sum_{r=0}^{\infty} \frac{\Gamma(r+p)}{\Gamma(p) \Gamma(r+1)} X_{r}(\cos \theta) z^{r},
$$

one has

$$
\frac{1}{(1-z)^{k+1}\left(1-2 z \cos \theta+z^{2}\right)^{p / 2}}=\sum_{n=0}^{\infty} S_{n}^{(k)} z^{n} .
$$

* L. Fejér, Über die Laplacesche Reihe, Mathematische Annalen, vol. 67 (1909), pp. 76-109; p. 81.

$\dagger \mathrm{S}$. Chapman, The general principle of summability, Quarterly Journal of Mathematics, vol. 43 (1912), pp. 1-52; p. 27.

$\ddagger$ Ibid., p. 45 . 
The generating function of the sequence $\left\{S_{n}^{(k)}\right\}$ is consequently

$$
\begin{aligned}
& \frac{1}{(1-z)^{k+1}\left(1-2 z \cos \theta+z^{2}\right)^{p / 2}} \\
& =\frac{1}{(1-z)^{k+1}\left(1-e^{i \theta} z\right)^{p / 2}\left(1-e^{-i \theta} z\right)^{p / 2}}
\end{aligned}
$$

This function may (for $0<\theta<\pi$ ) be developed into a power series in $z$ with unit radius of convergence. If $k+1>p / 2$, the predominant singularity on the circle of convergence is at $z=1$. Therefore, the leading term in the asymptotic expression of $S_{n}^{(k)}$ is given by the coefficient of $z^{n}$ in the expansion of

$$
\frac{1}{\left(1-e^{i \theta}\right)^{p / 2}\left(1-e^{-i \theta}\right)^{p / 2}(1-z)^{k+1}} \equiv \frac{1}{[2(1-\cos \theta)]^{p / 2}} \sum_{n=0}^{\infty} A_{n}^{(k)} z^{n} .
$$

Hence

$$
\begin{aligned}
S_{n}^{(k)} & =\frac{1}{[2(1-x)]^{p / 2}} A_{n}^{(k)}(1+o(1)), \quad(x=\cos \theta), \\
\lim _{n \rightarrow \infty} \frac{S_{n}^{(k)}}{A_{n}^{(k)}} & =\frac{1}{[2(1-x)]^{p / 2}}, \quad(-1<x<1),
\end{aligned}
$$

so that the series is summable $(C, k)$ for $k+1>p / 2$.

7. Second Application of Cesàro Summation to $R_{n}$. After application of summation $(C, i-1)$, the remainder terms (5) take the form (8), of which the highest ordered part is, from (9),

$$
\begin{aligned}
& (1 / 2) \Gamma(i) a_{n}(n+p)\left(X_{n+1}-X_{n}\right) \\
& =(1 / 2) \Gamma(i) a_{n} \frac{\Gamma(n+1)}{\Gamma(n+p)}(x-1) \sum_{r=0}^{n} \frac{\Gamma(r+p)}{\Gamma(r+1)}(2 r+p) X_{r} .
\end{aligned}
$$

From (10), if we let $t=1$, and make use of the equation (3) and the equation below (10), we find

$$
\begin{aligned}
& \sum_{r=0}^{\infty} \frac{\Gamma(r+p)}{\Gamma(r+1)}(2 r+p) X_{r}=\text { formal Cauchy product } \\
& \quad\left[\Gamma(p+1) \sum_{r=0}^{\infty} \frac{\Gamma(r+p)}{\Gamma(p) \Gamma(r+1)} X_{r}\right] \cdot\left[1+2 \sum_{r=1}^{\infty} \cos r v\right]
\end{aligned}
$$


and this is summable $(C, f)$ by Theorem 1 (combined with the above result of Chapman) to the value

$$
\frac{1}{[2(1-x)]^{p / 2}} \cdot 0=0
$$

where $f=(p / 2-1)+k+1>p / 2$, (for $k>0)$. Hence, from (11),

$$
\begin{gathered}
(1 / 2) \Gamma(i) a_{n} \frac{\Gamma(n+1)}{\Gamma(n+p)}(x-1) \sum_{r=0}^{n} \frac{\Gamma(r+p)}{\Gamma(r+1)}(2 r+p) X_{r} \\
=O\left(n^{(p-1) / 2+1-p}\right) o\left(n^{f}\right)=o\left(n^{f+(1-p) / 2}\right),
\end{gathered}
$$

by Theorem $4, f>p / 2$. Now apply summation $(C, j)$. The convergence factor multiplying $R_{n}^{(i-1)}$ is

$$
\frac{\Gamma(j+1)}{n^{j}}(1+o(1))
$$

so that the remainder terms (5) now become of order

$$
o\left(n^{j+(1-p) / 2}\right) O\left(n^{-j}\right)=o\left(n^{f+(1-p) / 2-j}\right)=o(1),
$$

if $f+(1-p) / 2-j \leqq 0$, that is, $j>1 / 2$.

Thus the two applications of Cesàro summability $(C, i-1)$ and $(C, j>1 / 2)$, which are seen to be equivalent to summability $(C, k>i-1 / 2)$ by Theorem 5 , cause the highest ordered part of the remainder terms (5), $R_{n}$, to approach zero as $n \rightarrow \infty$. The other parts of (8), being of lesser order, likewise approach zero by application of summability $(C, k>i-1 / 2)$. The value of $S_{\infty, i}^{(k>i-1 / 2)}$ can now be calculated as indicated in $\S 4$.

8. Legendre Polynomials. By letting $p=1$, one can easily obtain the values of $S_{\infty, i}^{(k)}$. In this case $a_{n}=1$ and $\sum_{0}^{\infty} X_{n}$ $=1 /(2-2 x)^{1 / 2}$. The remainder terms are handled by the use of Christoffel's formula

$$
\sum_{0}^{n}(2 i+1) X_{i}=(n+1) \frac{X_{n+1}-X_{n}}{x-1},
$$

the series $\sum_{0}^{\infty}(2 n+1) X_{n}$ having been previously proved summable $(C, k>1 / 2)$ to the value zero by Chapman.* The results obtained by this method check with those obtained by Brenke $\dagger$

* Chapman, loc. cit., p. 46.

$\uparrow$ Brenke, loc. cit. 
with the Hölder method of summability, where $i=1,2,3$. In the latter case, for example,

$$
\begin{aligned}
(x-1) S_{\infty, 3}^{(k)} & =2 S_{\infty, 1}^{(k)}+\frac{1}{4} S_{\infty, 0}^{(k)}+\frac{1}{3} \\
-\frac{1}{4} & {\left[(C, k) \text { of } \sum_{1}^{\infty} \frac{1}{(2 r-1)(2 r+3)} X_{r}\right], \quad(k>5 / 2) . }
\end{aligned}
$$

The University of Nebraska

\section{TRIANGULATION OF THE MANIFOLD OF CLASS ONE*}

BY S. S. CAIRNS

1. Introduction. In the present note, the writer shows that the triangulation method developed in an earlier paper $\dagger$ can be applied to divide a manifold of class one, as defined by Veblen and Whitehead, $\ddagger$ into the cells of a complex. The manifold of class one includes the regular $r$-manifold of class $C^{n}$ on a Riemannian space. $\S$

2. The Triangulation Theorem. Let $M_{r}$ be an arbitrary $r$ manifold of class one. A coordinate system is a correspondence between a point set, the domain of the system, on $M_{r}$, and a point set, called the arithmetic domain, in affine $r$-space. Allowable coordinate systems are a class of one-to-one correspondences whose properties are specified by axioms.|l

THEOREM. If an $r$-manifold, $M_{r}$, of class one is covered by the domains of a finite set of allowable coordinate systems, it can be triangulated into the cells of a finite complex. Otherwise it can be triangulated into the cells of an infinite complex.

* Presented to the Society, December 28, 1934.

$\dagger$ On the triangulation of regular loci, Annals of Mathematics, vol. 35 (1934), pp. 579-587. Hereafter we refer to this paper as Triangulations.

$\ddagger A$ set of axioms for differential geometry, Proceedings of the National Academy of Sciences, vol. 17 (1931), pp. 551-561; also, The Foundations of Differential Geometry, Cambridge Tract No. 29, 1932, Chapter 6, referred to below as Foundations.

$\S$ Marston Morse, The Calculus of Variations in the Large, Colloquium Publications of this Society, vol. 18 (1934), Chapter 5.

|| Veblen and Whitehead, loc. cit. 